\title{
Cálculo de algunas medidas estadísticas para evaluar el desempeño de redes Ad Hoc
}

\author{
Jorge E. Ortíz ${ }^{\S}$, Germán Hernández* \\ *Departamento de Ingeniería de Sistemas e Industrial, Universidad Nacional de Colombia \\ §e-mail: jeortiz@unal.edu.co
}

(Recibido: Abril 12 de 2005 - Aceptado: Julio 11 de 2006)

\begin{abstract}
Resumen
Para la realización del análisis de un protocolo de comunicaciones en una red de tipo Ad Hoc es necesario incluir en el modelo no solamente las características internas del protocolo sino, además, el contexto propio bajo el cual se desenvuelve. En la evaluación de su desempeño, en consecuencia, el protocolo debería ser probado en condiciones en las que se incluyan movimientos realistas de los usuarios móviles. En este artículo se revisan varios modelos de movilidad que fueron usados en la experimentación. Dado que resulta muy costoso realizar la evaluación sobre una red Ad Hoc real, se optó por una técnica basada en simulación que permitió extraer las conclusiones de esta investigación. Dentro de los modelos de movilidad usados se encuentran aquellos que representan nodos móviles cuyo movimiento es mutuamente independiente y modelos de movilidad que representan nodos móviles cuyos movimientos son interdependientes. Se presentan algunos resultados de la simulación en NS-2 que ilustran la importancia de escoger un modelo de movilidad en la simulación de un protocolo sobre redes móviles. Específicamente, se concluye que el desempeño de una red Ad Hoc depende del modelo de movilidad empleado y de sus parámetros.
\end{abstract}

Palabras Clave: Redes Ad Hoc, Modelo de movilidad, NS-2, Medida de desempeño, Protocolo de comunicación, Simulación.

\begin{abstract}
In the performance evaluation of a protocol for an Ad Hoc network, the protocol should be tested under realistic conditions including realistic movements of the mobile users. In this paper we revise several mobility models used in the simulations of mobile networks including mobility models that represent mobile nodes whose movements are independent of each other and mobility models that represent mobile nodes whose movements are dependent on each other. We present simulation results in NS-2 that illustrate the importance of choosing a mobility model in the simulation of a mobile network protocol. Specifically, we illustrate how the performance results of an Ad Hoc network protocol drastically change as a result of changing the mobility model simulated.
\end{abstract}

Keywords: Ad Hoc networks, Mobility models, NS-2, Performance measure, Communication protocol, Simulation.

\section{Introducción}

Este trabajo se desarrolla del siguiente modo. En la sección 1.1 se mencionan aplicaciones actuales de las redes Ad Hoc. En la sección 1.2 se presentan conceptos sobre simulación de redes Ad Hoc. En la sección 2 se discuten cuatro modelos sintéticos de movilidad incluyendo movilidad en grupos y de

entidades. En la sección 3 se describe el esquema de experimentación empleado para obtener los resultados. En la sección 4 se discute cómo un modelo de movilidad tiene un efecto importante en la evaluación del desempeño de un protocolo de redes Ad Hoc. Se termina el artículo con una 
discusión sobre los resultados experimentales más importantes y se esbozan desarrollos futuros.

\subsection{Definición de redes Ad Hoc}

Las redes inalámbricas se pueden clasificar en dos tipos: primero redes con infraestructura que presentan gateways fijos cableados (Tanenbaum, 1996), un nodo móvil se comunica con un punto en la red, llamado estación base, dentro de su radio de comunicación. El nodo móvil se puede mover geográficamente mientras se esté comunicando con su estación base dentro de su radio de comunicación. Cuando sale del rango de una estación base el nodo móvil se conecta con una nueva estación base fija y comienza la comunicación a través de ésta. Este proceso se conoce como handoff y se presenta en varios sistemas de comunicación, especialmente los sistemas celulares (The CMU Monarch Project, 2002).

El segundo tipo de redes son las denominadas redes Ad Hoc o sin estructura. En las redes Ad Hoc todos los nodos son móviles y pueden ser conectados dinámicamente de manera arbitraria (Broch et al., 1998). Todos los nodos de estas redes se comportan como enrutadores y participan en el descubrimiento y mantenimiento de rutas a otros nodos de la red.

La figura 1 muestra una red Ad Hoc simple en la que el nodo móvil A desea enviar un paquete al nodo móvil $\mathrm{C}$ pero $\mathrm{C}$ está fuera del rango, de esta manera al nodo móvil $\mathrm{B}$ se le pasa el paquete proveniente de A y este, a su vez, lo transmite hacia C. El ejemplo ilustra claramente que las redes Ad Hoc consisten en hosts inalámbricos que se comunican unos con otros en la ausencia de una infraestructura fija.

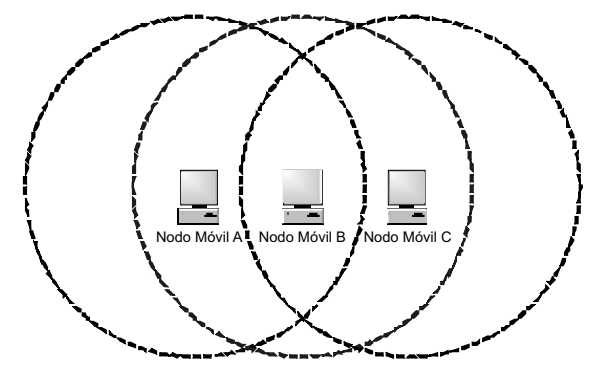

Figura 1. Red Ad Hoc simple
Las rutas entre dos nodos en una red Ad Hoc pueden consistir en saltos a través de otros hosts en la red. La movilidad de los nodos puede causar cambios impredecibles en la topología de la red, por consiguiente encontrar y mantener rutas es una de las tareas más importantes en los protocolos de enrutamiento (Broch et al., 1998).

\subsection{Simulación de redes Ad Hoc}

Los principales parámetros que afectan el desempeño de una red Ad Hoc son: número de nodos móviles en un área, comportamiento de los nodos móviles (movimiento) y calidad de los enlaces, esto puede verse más ampliamente en (Camp et al., 2002) y (Chiang, 1998).

Para poder simular correctamente un nuevo protocolo para una red Ad Hoc, es necesario usar un modelo de movilidad que represente los nodos móviles (NM) que eventualmente utilizarán el protocolo dado. Solamente en este tipo de escenario simulado es posible determinar si el protocolo propuesto será útil cuando se implemente. Actualmente existen dos tipos de modelos de movilidad usados en simulación de redes: modelos que utilizan rastros y modelos sintéticos. Los modelos que utilizan rastros son aquellos cuyos datos a analizar son tomados de sistemas reales; éstos proveen información exacta especialmente cuando involucran un número largo de participantes y un largo periodo de observación. Sin embargo, ambientes nuevos de redes (como por ejemplo, redes Ad Hoc) no son fácilmente modelados si los rastros no han sido creados, caso en el cual se deben utilizar modelos sintéticos. Los modelos sintéticos intentan representar de manera realista el comportamiento de nodos móviles sin el uso de rastros. En este artículo se presentan cuatro modelos sintéticos que han sido propuestos para la evaluación de protocolos de redes Ad Hoc.

Un modelo de movilidad debe intentar imitar los movimientos reales de nodos móviles. Los cambios de velocidad y dirección deben existir en instantes razonables de tiempo. No es deseable, por ejemplo, que los nodos móviles viajen en una línea recta con velocidad constante durante toda la simulación ya que un nodo móvil real no viajaría 
de esa manera tan restringida.

Se revisa un número importante de modelos sintéticos usados en la simulación de redes Ad Hoc. Los detalles de estos modelos proveen un buen recurso para investigadores y diseñadores de redes. Adicionalmente implementaciones de todos los modelos de movilidad descritos en este artículo están disponibles en (Ortiz, 2006).

\section{Ambientes de movilidad para los nodos de la red}

\subsection{Modelos de movilidad}

En esta sección se presentan cuatro modelos de movilidad que han sido propuestos o usados en la evaluación de desempeño de protocolos en redes Ad Hoc y que fueron implementados para desarrollar el estudio. Los primeros tres modelos presentados, el modelo Random Waypoint, el modelo Gauss-Markov y el modelo en ciudades, son representaciones de movilidad para entidades mientras que el modelo de movilidad de grupo con punto de referencia se refiere a movimiento de grupos de entidades. Detalles más amplios sobre estos modelos de movilidad se presentan en (Chiang, 1998; Chiang \& Gerla, 1998) y en (Davies, 2000).

\subsection{Modelo de movilidad Random Waypoint}

El modelo de movilidad Random Waypoint incluye pausas entre cambios de dirección y/o velocidad.

\section{RANDOM WAY POINT}

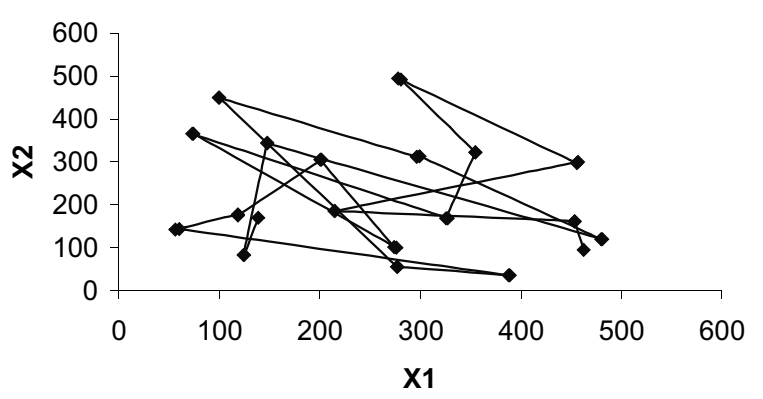

Figura 2. Ejemplo del patrón de movimiento del modelo Random Waypoint

Un nodo móvil comienza en una localización en un determinado periodo de tiempo, es decir una pausa. Una vez que el tiempo expira, el nodo móvil escoge un destino aleatorio y una velocidad uniformemente distribuida. Luego el nodo móvil viaja hacia el nuevo destino con la velocidad seleccionada. Cuando llega el nodo móvil hace una pausa en un periodo específico de tiempo antes de empezar de nuevo el proceso. En la Figura 2 se muestra como es el patrón de movimiento del modelo Randowm Waypoint.

\subsection{Modelo de movilidad Gauss-Markov}

El modelo de movilidad Gauss-Markov fue originalmente propuesto para la simulación de PCS. Sin embargo, este modelo ha sido usado también para la simulación de redes Ad Hoc. En esta sección se describe una implementación del modelo.

El modelo de movilidad de Gauss-Markov fue diseñado para adaptarse a varios niveles de aleatoriedad por medio del ajuste de un parámetro. Inicialmente a cada nodo NM se le asigna una dirección y una velocidad. En intervalos fijos de tiempo $t$, el movimiento ocurre actualizando la velocidad y la dirección de cada nodo móvil. Específicamente, los valores de la velocidad y dirección en el tiempo $t$ se calculan con base en los valores de la velocidad y dirección en el tiempo $t$ - 1 mediante las ecuaciones (1) y (2) usando una variable aleatoria $\alpha$ :

$$
\begin{gathered}
s_{t}=\alpha s_{t-1}+(1-\alpha) s+\sqrt{\left(1-\alpha^{2}\right) s_{x_{t-1}}} \\
d_{t}=\alpha d_{t-1}+(1-\alpha) d+\sqrt{\left(1-\alpha^{2}\right) d_{x_{t-1}}}
\end{gathered}
$$

donde $s_{t}$ y $d_{t}$ son la nueva velocidad y dirección de los nodos móviles en el intervalo de tiempo $t, \alpha \quad$ (con $0 \leq \alpha \leq 1$ ) es el parámetro de ajuste usado para variar la aleatoriedad, y $s$ y $d$ son constantes que representan el valor de la media de la velocidad y la dirección cuando $t \rightarrow \infty$ y $s_{x_{t-1}}, d_{x_{t-1}}$ son variables aleatorias de una distribución normal estándar. Valores totalmente aleatorios se obtienen haciendo $\alpha=0$ y movimiento lineal se obtiene con $\alpha=1$. Niveles intermedios de aleatoriedad se obtienen variando el valor de $\alpha$ entre 0 y 1 . En la figura 3 se muestra el patrón de movimiento de un nodo en el modelo de movilidad Gauss-Markov. 


\section{GAUSS MARKOV}

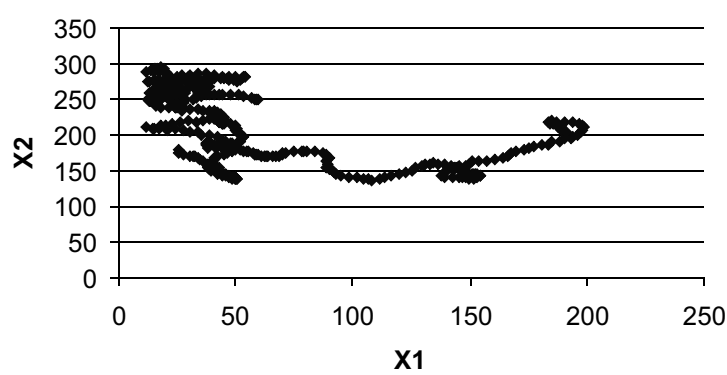

Figura 3. Patrón de movimiento de un nodo en el modelo de movilidad Gauss-Markov

\subsection{Modelo de movilidad en ciudad}

En el modelo de movilidad de ciudad, el área de la simulación es una red de calles que representan una parte de una ciudad donde existe una red Ad Hoc. Las calles y los límites de velocidad están basados en el tipo de ciudad que está siendo simulada. Por ejemplo, las calles pueden formar una rejilla en el centro de la ciudad con una autopista cerca al borde de la simulación para representar una avenida que rodea la ciudad.

Cada nodo móvil empieza la simulación en un punto definido en alguna calle. Luego de la iniciación un nodo móvil escoge un lugar representado por un punto en una calle. El algoritmo que define el movimiento de la ubicación localiza una ruta correspondiente al camino más corto entre los dos puntos $\mathrm{y}$ adicionalmente características como límite de velocidad y distancia mínima permitida entre dos nodos móviles (Chiang, 1998).

\section{MOVILIDAD EN CIUDADES}

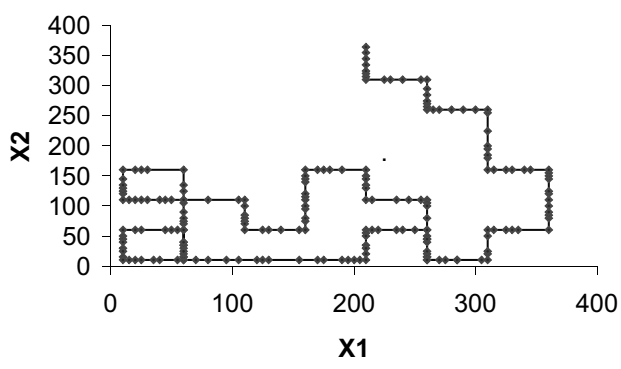

Figura 4. Patrón de movimiento de un nodo en el modelo de movilidad en ciudades
Cuando el nodo llega a su destino, el nodo móvil se detiene por un tiempo específico, luego elige aleatoriamente otra ubicación dentro de la ciudad y comienza el proceso de nuevo. En la figura 4 se muestra el patrón de movimiento de un nodo en el modelo de movilidad en ciudades.

\subsection{Modelo de movilidad de grupo con punto de referencia}

El modelo de movilidad con punto de referencia representa el movimiento aleatorio de un grupo de nodos móviles así como el movimiento aleatorio de cada nodo móvil dentro del grupo. Los movimientos de grupo son basados en el camino que recorre un centro lógico del grupo. El centro lógico del grupo se calcula usando un vector de movimiento de grupo, GM. El movimiento del centro del grupo caracteriza completamente el movimiento de su grupo correspondiente incluyendo su dirección y velocidad. Los nodos móviles individuales se mueven de manera aleatoria alrededor de sus propios puntos de referencia predefinidos, cuyos movimientos dependen del movimiento del grupo. Tan pronto como los puntos individuales de referencia se mueven de un tiempo al siguiente sus ubicaciones se actualizan de acuerdo con un centro lógico (Davies, 2000).

El modelo RPGM, como se conoce este modelo, fue diseñado para ilustrar escenarios como rescates de avalanchas. Durante un rescate de avalancha el equipo de rescate consiste en humanos y caninos que trabajan de manera cooperativa. Los guías humanos tienden a mostrar un camino para que los caninos los sigan, ya que estos conocen la ubicación aproximada de las víctimas. Los perros crean sus propios caminos aleatorios alrededor del área general que es escogida por los humanos. Este modelo se definió originalmente en (Basagni et al., 1998).

\section{Diseño experimental}

En esta sección se muestra, a través de simulación, la importancia de escoger el modelo de movilidad en el momento de evaluar el desempeño de una redAdHoc. 
Se usa NS-2 (The network simulator, 2002) para comparar el desempeño de los cuatro modelos descritos anteriormente: Modelo de movilidad de grupo con punto de referencia, en ciudad, GaussMarkov, Random Waypoint, por medio de simulación de 100 nodos móviles. El conjunto de simuladores, datos resultantes, programas construidos para el análisis de los datos y otros resultados que, por espacio, no se han incluido aquí, se encuentran disponibles en la página web referenciada en (Ortiz, 2006).

Cada nodo móvil tiene $100 \mathrm{~m}$ de rango de transmisión y el enrutamiento de los paquetes se realiza a través de DSR. Los parámetros de los cuatro modelos de movilidad estudiados se escogieron de tal forma que la simulación de las rutas fuera lo más real posible y los modelos pudieran ser comparados unos con otros (Bar-Noy et al., 1994).

DSR es un protocolo de origen que determina rutas sobre demanda, en un protocolo de origen cada paquete lleva la ruta completa (una secuencia con una lista de nodos) que el paquete debe atravesar para llegar a su lugar de destino. En un protocolo sobre demanda o reactivo como el caso de DSR, una ruta a un destino se requiere solo cuando hay datos para enviar a un destino y la ruta a ese destino es desconocida o ha expirado. Se escogió DSR debido a que se comporta de manera adecuada en las evaluaciones de desempeño de protocolos unicast.

Parte del código de NS-2 que se usó en las simulaciones fue obtenido en (Boleng, 2001), aunque, en gran medida, fue desarrollado en los laboratorios de la Universidad Nacional. Las simulaciones se ejecutaron para $2010 \mathrm{seg}$. Sin embargo, se recolectaron $1010 \mathrm{seg}$ de tiempo de simulación debido a las razones expuestas.

En las simulaciones se tienen 20 fuentes CBR (constant bit rate) enviando paquetes a una tasa de un paquete por segundo a 20 receptores distintos es decir una simulación donde son transmitidos 20,000 paquetes entre 20 parejas de comunicación. Todos los resultados de desempeño se presentan en un promedio de 5 simulaciones, las localizaciones iniciales de los nodos móviles son aleatorias y se distribuyen uniformemente, esto puede verse mejor en (Garcia-Luna-Aceves \& Madrga, 1999).

En la comparación de los cuatro modelos de movilidad, se consideran las siguientes métricas de desempeño: razón de entrega de paquetes de datos sobre paquetes de actualización de rutas, la razón del número de paquetes que se mandan sobre el número de paquetes que se reciben, número promedio de saltos, y demora de arribo de paquetes.

\section{Análisis de resultados}

A continuación se presentan los resultados comparativos de las simulaciones para cada una de las medidas de desempeño en intervalos de 100 seg. En las gráficas, cada intervalo se representa por un número entre 1 y 10 . Así, por ejemplo, el número 3 significa un intervalo entre 200 y 300 seg. Fácilmente se deduce que, como se había supuesto al principio, el modelo de movilidad afecta directamente el desempeño del protocolo.

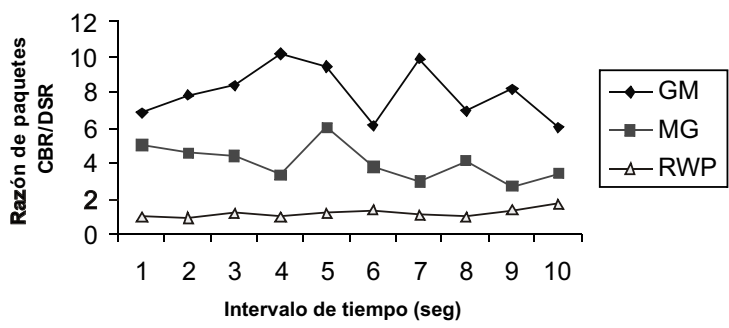

Figura 5. Comparación de modelos con paquetes de $64 \mathrm{~kb}$ en cuanto a la razón de paquetes de datos sobre paquetes de enrutamiento

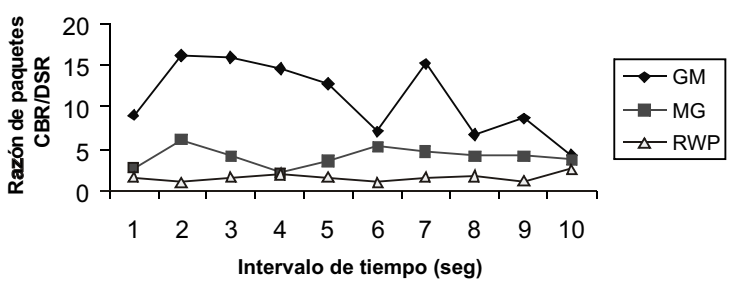

Figura 6. Comparación de modelos con paquetes de $512 \mathrm{~kb}$ en cuanto a la razón de paquetes de datos sobre paquetes de enrutamiento 


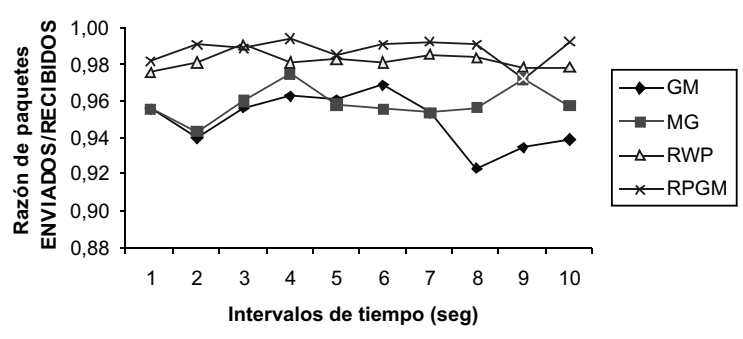

Figura 7. Comparación de modelos con paquetes de $64 \mathrm{~kb}$ en cuanto a la razón de paquetes de enviados sobre recibidos

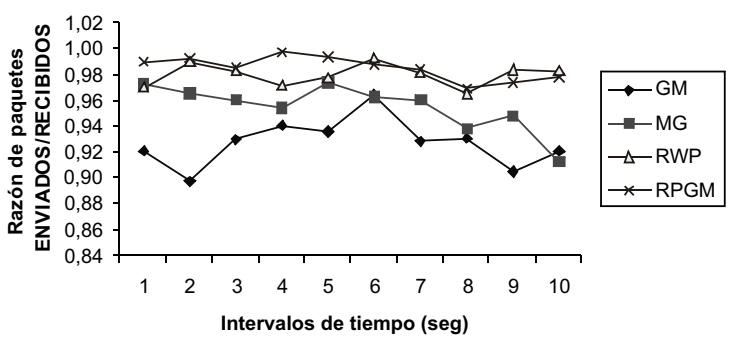

Figura 8. Comparación de modelos con paquetes de $512 \mathrm{~kb}$ en cuanto a la razón de paquetes de enviados sobre recibidos

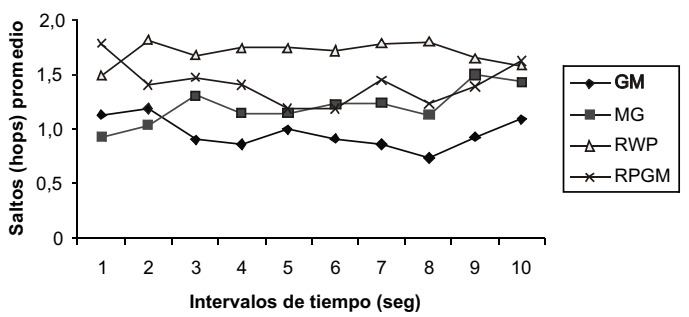

Figura 9. Comparación de modelos con paquetes de $64 \mathrm{~kb}$ en cuanto al número promedio de saltos

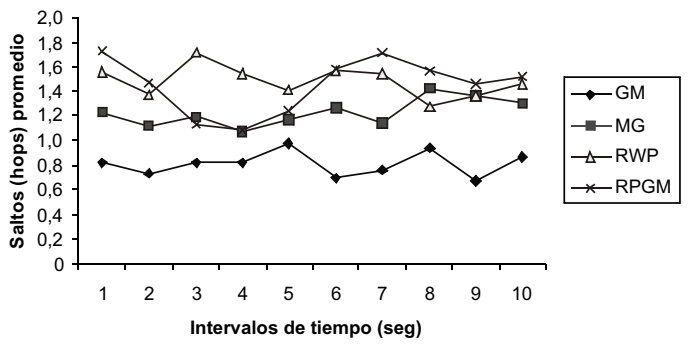

Figura 10. Comparación de modelos con paquetes de $512 \mathrm{~kb}$ en cuanto al número promedio de saltos

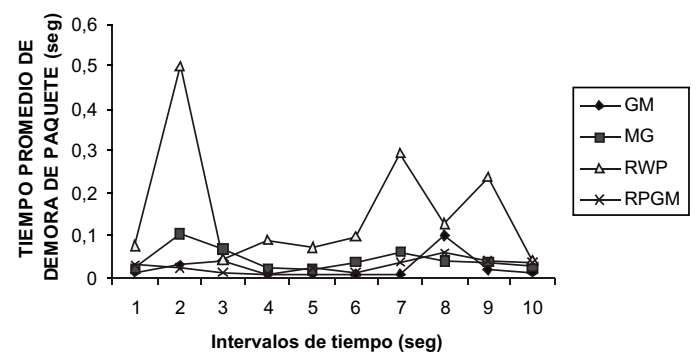

Figura 11. Comparación de modelos con paquetes de $64 \mathrm{~kb}$ en cuanto al tiempo promedio de demora de paquete

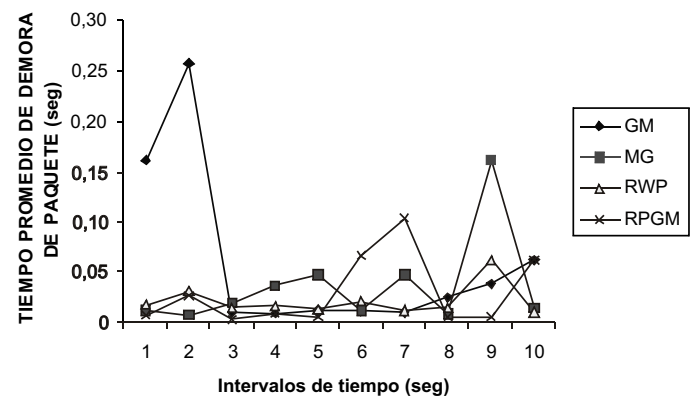

Figura 12. Comparación de modelos con paquetes de $512 \mathrm{~kb}$ en cuanto al tiempo promedio de demora de paquete

\section{Conclusiones}

Del estudio se desprenden las siguientes conclusiones, dificultades y desarrollos futuros:

A). El desempeño de un protocolo en una red Ad Hoc puede variar significativamente bajo diferentes modelos de movilidad de un protocolo RTP. Las figuras 5 a 12 ilustran el desempeño de un protocolo de enrutamiento con diferentes modelos de movilidad. Es claro que el desempeño del protocolo se afecta de manera significativa por el modelo escogido.

B). El desempeño de una red Ad Hoc puede variar significativamente cuando el mismo modelo de movilidad se usa con diferentes parámetros. Esto se ilustra en las figuras 11 a 12 donde se simula el mismo modelo de movilidad con un tamaño diferente de paquetes.

C). El desempeño del protocolo de una red Ad Hoc debería ser evaluado con el modelo de movilidad que sea más parecido al escenario real que se espera. El hecho de conocer el escenario 
protocolo para red Ad Hoc. En caso de que el escenario real no sea conocido, los investigadores deben hacer una elección apropiada acerca del modelo de movilidad que se podría utilizar.

D). Se evidencia la importancia en la investigación sobre modelos de movilidad para protocolos en redes Ad Hoc. Una dirección para la investigación es examinar movimientos de los nodos móviles en escenarios reales para producir modelos de movilidad más precisos, otra dirección seria combinar las mejores características de modelos de movilidad para crear un modelo nuevo.

E). Dentro del desarrollo futuro se espera investigar el desempeño de aplicaciones especificas en redes Ad Hoc. Por ejemplo, correo electrónico, transferencia de archivos y aplicaciones multimedia. Se espera, igualmente, poder realizar simulaciones en áreas geográficas más amplias con un mayor número de nodos. Es también interesante evaluar el desempeño de los protocolos en escenarios integrados de redes cableadas y redes inalámbricas.

F). A medida que el modelo imita mejor el sistema real, su complejidad aumenta al igual que los requerimientos computacionales.

\section{Referencias bibliográficas}

Bar-Noy, A., Kessler, I., \& Sidi, M. (1994). Mobile users: to update or not to update? In Proceedings of the Joint Conference of the IEEE Computer and Communications Societies (INFOCOM), p. 570 - 576.

Basagni, S., Chlamtac, I., Syrotiuk, V.R., \& Woodward, B.A. (1998). A distance routing effect algorithm for mobility (DREAM). In Proceedings of the IEEE/ACM International Conference on Mobile Computing and Networking (MOBICOM), p. 76-84.

Boleng J. (2001). Normalizing mobility characteristics and enabling adaptive protocols for Ad Hoc networks. In Proceedings of the Local and Metropolitan Area Networks Workshop (LANMAN), p. 9-12.
Broch, J., Maltz, D.A., Johnson, D.B., Hu,Y.C., \& Jetcheva, J. (1998). A performance comparison of multi-hop wireless ad hoc network routing protocols. In Proceedings of the IEEE/ACM International Conference on Mobile Computing and Networking (MOBICOM), p. 85-97.

Camp,T., Boleng, J., Williams, B., Wilcox, L., \& Navidi,W. (2002). Performance evaluation of two location based routing protocols. In Proceedings of the Joint Conference of the IEEE Computer and Communications Societies (INFOCOM), p. 16781687.

Chiang, C.C. (1998). Wireless network multicasting. PhD Thesis, University of California, Los Angeles.

Chiang, C.C., \& Gerla, M. (1998). On-demand multicast in mobile wireless networks. In Proceedings of the IEEE International Conference on Network Protocols (ICNP), p. 262.

Davies, V. (2000). Evaluating mobility models within an Ad Hoc network. Master's Thesis, Colorado School of Mines.

Garcia-Luna-Aceves, J.J., \& Madrga, E.L. (1999). A multicast routing protocol for ad-hoc networks. In Proceedings of the Joint Conference of the IEEE Computer and Communications Societies (INFOCOM), p. 784-792.

Garcia-Luna-Aceves, J.J., \& Spohn, M. (1999). Source-tree routing in wireless networks. In Proceedings of the 7th International Conference on Network Protocols (ICNP), p. 273-282.

Ortiz, J . ( ( $\left.\begin{array}{llll}2 & 0 & 0 & 6\end{array}\right)$. W e b p a g e : http://dis.unal.edu.co/profesores/jortiz

Tanenbaum, A.S. (1996). Computer networks. Prentice-Hall, Inc.

The CMU Monarch Project. (2002). The CMU monarch extensions to the NS simulator. http://www.monarch.cs.cmu.edu/

The network simulator (2002). NS-2. http://www.isi.edu/nsnam/ns/ 